\title{
MOTION-COMPENSATED COMPRESSED-SENSING RECONSTRUCTION FOR DYNAMIC MRI
}

\author{
Sungkwang Mun and James E. Fowler \\ Department of Electrical and Computer Engineering, Geosystems Research Institute, \\ Mississippi State University, USA
}

\begin{abstract}
Compressed-sensing reconstruction using motion estimation and compensation for dynamic MRI data is proposed. Reconstruction is driven from a residual in the $k$-space domain between the current-frame measurements and a corresponding motion-compensated prediction. Due to the periodicity commonly exhibited in dynamic MRI, a telescopic motion search through the entire group of pictures is used to determine the best match for the block-based motion estimation. Experimental comparisons demonstrate improved performance as compared to existing dynamic-MRI reconstructions, both those with and without motion compensation.
\end{abstract}

Index Terms - compressed sensing, dynamic MRI

\section{INTRODUCTION}

Recent theoretical and practical advances in compressed sensing (CS) of images and video has spawned interest in applying CS methodology to sequences of magnetic resonance imagery (MRI). Such temporally varying imagery is commonly referred to as dynamic MRI, and several algorithms have been devised specifically for its reconstruction from CS measurements, e.g., [1-5]. While CS-based sampling has the potential to greatly accelerate the acquisition process of dynamic-MRI data, by capitalizing on methodologies such as motion estimation (ME) and motion compensation (MC) that arise in conventional video processing, we can exploit temporal redundancy existing in dynamic MRI to improve CS reconstruction. Specifically, in this paper, we propose a CS reconstruction for dynamic MRI that is inspired by an ME/MCbased CS framework that we developed previously for conventional video [6-8]. That is, given a collection of subsampled kspace (frequency-space) measurements, we reconstruct a projected residual that is the difference between the target frame and a prediction of the frame in the space of the frequency measurements. In order to obtain the prediction, we employ block matching using a telescopic search that looks into the several previously reconstructed frames. The combination of these techniques provides greatly enhanced reconstruction quality as compared to a simple "intraframe," or frame-by-frame, reconstruction.

There have been several prior algorithms for the CS reconstruction of dynamic MRI; some of these employ ME/MC, while others do not. A prominent example of a technique that employs $\mathrm{ME} / \mathrm{MC}$ is the k-t FOCUSS algorithm of $[1,2]$. In k-t FOCUSS, it is assumed that there exist key frames (obtained through some separate means) such that CS reconstruction is driven by MC residuals between each intervening non-key frame and a block-based bidirectional ME/MC prediction from each of the nearest key frames. On the other hand, a prominent example of a technique that does not employ ME/MC is [3] wherein a strategy of finding the $\ell_{1}$ optimal solution outside the currently known sparsity pattern (which is tracked frame to frame) was proposed under the name "ModifiedCS." Subsequently, this framework was combined with a residual reconstruction as "Modified-CS-Residual" in [9]. The assumption underlying these latter two methods is that the temporal evolution of the sparsity pattern is slow, which is indeed true for static parts of dynamic MRI data. The key difference between residual reconstruction in Modified-CS-Residual and the ME/MC-based strategies such as k-t FOCUSS is that Modified-CS-Residual obtains the prediction using an explicit sparsity pattern rather than using $\mathrm{ME} / \mathrm{MC}$-based prediction based on a temporal search.

The contributions of the present paper include 1) the development of a fast yet effective 2D reconstruction for MRI using a projected Landweber (PL) [10] framework wherein a directional transform and statistical thresholding are incorporated; and 2) the incorporation into this PL reconstruction of ME/MC based on a telescopic search that aids block matching and results in improved residual reconstruction for dynamic MRI. We refer to the resulting algorithm as motion-compensated CS with PL reconstruction (MC-CS-PL). We note that, despite some similarities, there exist substantial differences between MC-CS-PL and the k-t FOCUSS techniques of $[1,2]$. Most saliently, our proposed MC-CS-PL is built on frame-to-frame ME/MC in which all frames participate as reference frames for ME/MC, instead of driving ME/MC exclusively from (potentially distant) key frames as in k-t FOCUSS. Also, the telescopic search in our proposed method results in a greater likelihood of finding an accurate matching block since the search is conducted over multiple frames.

\section{BACKGROUND}

The CS paradigm concerns the reconstruction of a sparse (or compressible) signal $\mathbf{x}$ with length $N$ from $M$ measurements $\mathbf{y}=\mathbf{\Phi} \mathbf{x}$ where $M \ll N, \boldsymbol{\Phi}$ is an $M \times N$ measurement matrix, and $S=$ $M / N$ is subsampling ratio or subrate. For MRI data, a partial Fourier matrix is commonly used for $\boldsymbol{\Phi}$ (e.g., Fig. 8 in [11]).

One effective method for CS reconstruction of $\mathbf{x}$ from measurements $\mathbf{y}$ is a PL algorithm [10,12-14], which has also been called "iterative thresholding" (e.g., $[15,16])$. PL starts from some initial approximation $\check{\mathbf{x}}^{(0)}$ and forms the approximation at iteration $i+1$ as

$$
\begin{aligned}
\check{\check{\mathbf{x}}}^{(i)} & =\check{\mathbf{x}}^{(i)}+\frac{1}{\gamma} \boldsymbol{\Psi} \boldsymbol{\Phi}^{T}\left(\mathbf{y}-\boldsymbol{\Phi} \boldsymbol{\Psi}^{-1} \check{\mathbf{x}}^{(i)}\right), \\
\check{\mathbf{x}}^{(i+1)} & = \begin{cases}\check{\check{\mathbf{x}}}^{(i)}, & \left|\check{\mathbf{x}}^{(i)}\right| \geq \tau^{(i)} \\
0 & \text { else. }\end{cases}
\end{aligned}
$$

Here, $\gamma$ is a scaling factor ([13] uses the largest eigenvalue of $\left.\boldsymbol{\Phi}^{T} \boldsymbol{\Phi}\right)$, while $\tau^{(i)}$ is a threshold set appropriately at each iteration. Advantages of PL-based CS reconstruction include reduced computational complexity along with flexibility of choosing a sparsity transform $\Psi$ as well as the thresholding method. 

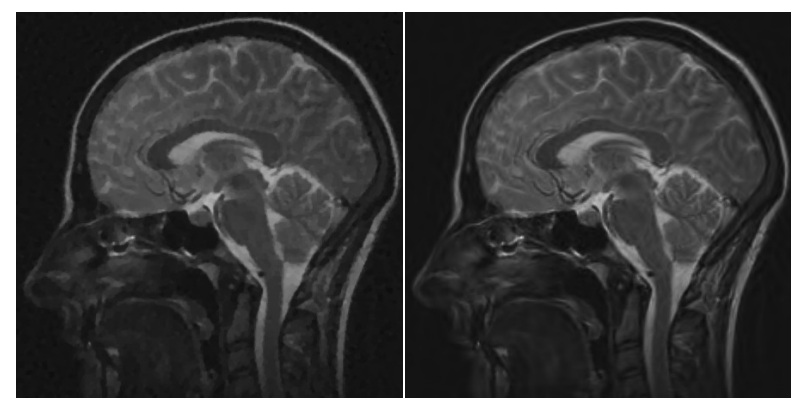

Figure 1: Sagittal brain image $(450 \times 450)$, subrate $S=0.17$. Left: TV (37 dB, 39 min), Right: CS-PL (39 dB, $92 \mathrm{sec}$ )

\section{MOTION-COMPENSATED CS FOR DYNAMIC MRI}

\subsection{PL with Directional Transforms}

The PL algorithm based on (1) and (2) and designed for MRI is as follows:

$$
\begin{aligned}
& \text { function } \mathbf{x}^{(i+1)}=\operatorname{PL}\left(\mathbf{x}^{(i)}, \mathbf{y}, \boldsymbol{\Phi}, \boldsymbol{\Psi}, \lambda\right) \\
& \check{\mathbf{x}}^{(i)}=\mathbf{\Psi} \mathbf{x}^{(i)} \\
& \check{\mathbf{x}}^{(i)}=\operatorname{Threshold}\left(\check{\mathbf{x}}^{(i)}, \lambda\right) \\
& \overline{\mathbf{x}}^{(i)}=\boldsymbol{\Psi}^{-1} \check{\mathbf{x}}^{(i)} \\
& \mathbf{x}^{(i+1)}=\overline{\mathbf{x}}^{(i)}+\boldsymbol{\Phi}^{T}\left(\mathbf{y}-\mathbf{\Phi} \overline{\mathbf{x}}^{(i)}\right)
\end{aligned}
$$

Here, Threshold $(\cdot)$ is a thresholding process as discussed below. In our use of PL, we initialize with $\mathbf{x}^{(0)}=\boldsymbol{\Phi}^{T} \mathbf{y}$ and terminate when $\left|D^{(i+1)}-D^{(i)}\right|<10^{-4}$, where $D^{(i)}=\frac{1}{\sqrt{N}} \| \mathbf{x}^{(i)}-$ $\mathbf{x}^{(i-1)} \|_{2}$. For $\boldsymbol{\Psi}$, we use a dual-tree discrete wavelet transform (DDWT) [17], the effectiveness for CS reconstruction of which was demonstrated in [10]. For thresholding, we apply bivariate shrinkage [18] on the DDWT coefficients as was done in [10]. Specifically, for each coefficient of $\check{\mathbf{x}}^{(i)}$,

$$
\operatorname{Threshold}(\check{\check{x}}, \lambda)=\frac{\left(\sqrt{\check{\check{x}}^{2}+\check{\check{x}}_{p}^{2}}-\lambda \frac{\sqrt{3} \sigma^{(i)}}{\sigma_{\check{x}}}\right)_{+}}{\sqrt{\check{\check{x}}^{2}+\check{\check{x}}_{p}^{2}}} \cdot \check{\check{x}},
$$

where $(g)_{+}=0$ for $g<0,(g)_{+}=g$ else; $\check{x}_{p}$ is the coefficient in the parent decomposition level; $\sigma^{(i)}$ and $\sigma_{\check{x}}$ are noise and signal variance, respectively; and $\lambda$ is a convergence-control factor.

We refer to the overall process-which will form the foundation of the dynamic-MRI reconstruction discussed next-as CSPL. Example reconstruction of CS-PL is compared to well-known TV reconstruction (e.g., [19]) in Fig. 1 for a single (2D) MRI image. While the visual quality is similar, CS-PL is around 20 times faster than $\mathrm{TV}^{1}$. We consequently use CS-PL as the base reconstruction in the sequel.

\subsection{Residual Reconstruction}

Based on the assumption that MR images have high temporal correlation, one frame can be enhanced by the information from the previously reconstructed frames. Suppose we have measurements, $\mathbf{y}$, of the current frame, $\mathbf{x}$, and its prediction $\mathbf{y}_{\text {pred }}$ where the latter is obtained by a $\mathrm{ME} / \mathrm{MC}$ process using previously reconstructed

\footnotetext{
${ }^{1}$ Here, we use the $\ell_{1}$-MAGIC implementation (http://www. 11-magic.org); although there are faster TV-based reconstructions (e.g., [20]), we have observed that the $\ell_{1}$-MAGIC implementation achieves better reconstruction quality.
}

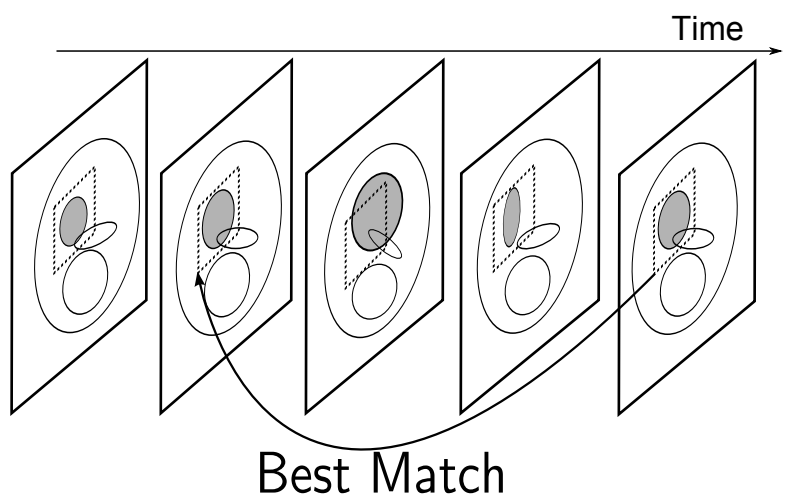

Figure 2: Telescopic search over a GOP in dynamic MRI. The dotted box in the last frame (current frame) represents the current block. The best matches in all the other frames to the end of the GOP are found (the dotted boxes in the other frames); the overall best match (in this case, in the second frame) is chosen as the prediction for the current block.

frame(s). Instead of a straightforward 2D reconstruction using $\mathbf{y}$, we can reconstruct the projected residual of the measurements to exploit temporal correlation; i.e.,

$$
\mathbf{y}_{\mathrm{r}}=\mathbf{y}-\boldsymbol{\Phi} \hat{\mathbf{x}}_{\text {pred }} .
$$

It is clear that $\mathbf{y}_{\mathbf{r}}$ is the projection of the residual, $\mathbf{x}_{\mathrm{r}}$, between our prediction $\hat{\mathbf{x}}_{\text {pred }}$ and the original and still-unknown $\mathbf{x}$; i.e.,

$$
\mathbf{y}_{\mathrm{r}}=\mathbf{y}-\boldsymbol{\Phi} \hat{\mathbf{x}}_{\text {pred }}=\boldsymbol{\Phi}\left(\mathbf{x}-\hat{\mathbf{x}}_{\text {pred }}\right)=\boldsymbol{\Phi} \mathbf{x}_{\mathrm{r}} .
$$

If the prediction process is accurate, the residual frame $x_{\mathrm{r}}$ should be more compressible than the original frame $\mathbf{x}$, so its reconstruction should be more accurate. Consequently, we can form a new approximation to $\mathbf{x}$ as

$$
\hat{\mathbf{x}}=\hat{\mathbf{x}}_{\text {pred }}+\hat{\mathbf{x}}_{\mathrm{r}}
$$

This process is called residual reconstruction (e.g., [2, 6-9]). We now have a new approximation to the current frame that is of better quality than the initial approximation that we created from a direct CS-PL reconstruction from $y$. We turn our attention to the issue of producing the prediction of the current frame next.

\section{3. $\mathrm{ME} / \mathrm{MC}$ with Telescopic Search}

In traditional video processing, $\mathrm{ME} / \mathrm{MC}$ is used to reduce temporal redundancy by tracking object motion from frame to frame. ME is applied between two frames to estimate a motion field, and such ME could be performed in a variety of ways. For simplicity, we consider full-search, block-based ME. Consequently, the initial reconstruction of the current frame is partitioned into blocks whose motion from a reference frame is determined by a block-matching search. We note that we group a number of consecutive frames together as a group of pictures (GOP) as is commonly done in traditional video processing, and the motion search is performed within the GOP. The first frame of the GOP is denoted as a "key frame" which typically has a higher subrate than the other "nonkey frames" of the GOP.

We have previously employed ME/MC for the reconstruction of conventional video from CS measurements (e.g., [6-8]). In conventional video, block-based $\mathrm{ME} / \mathrm{MC}$ performs well when objects 
undergo geometric change (i.e., translation) from one frame to the next. In case of certain dynamic-MRI sequences, a motion search using only the immediately preceding frame is insufficient to find a good match which might exist in the sequence long before. An example of such a sequence is the dynamic MRI of a heart, wherein contractions and expansions of a cardiac chamber repeat at a certain interval; in this case, the best match for the current block might lie a number of frames previous, depending on the frequency of the cardiac pulsations.

More effective block matching in sequences with repetitive patterns can be obtained by searching over several previous frames using a telescopic search [21-23], illustrated in Fig. 2 for dynamic MRI. The previous frame is used as the reference for the usual block search for the current block in the current frame. The location of the resulting best-matching block in the reference frame is then in turn used as the start location for a motion search in the immediately preceding frame, and this process is repeated until the end of the GOP is reached. Then, the best match from any of the searched frames becomes the final prediction.

We extend the CS-PL of the previous section by incorporating telescopic ME/MC, resulting in the MC-CS-PL algorithm that reconstructs the current frame at time $t$ :

$$
\begin{aligned}
& \text { function } \hat{\mathbf{x}}_{t}=\operatorname{MC}-\operatorname{CS}-\operatorname{PL}\left(\mathbf{y}_{t}, \boldsymbol{\Phi}, \mathbf{\Psi}, \hat{\mathbf{x}}_{t-1}, \hat{\mathbf{X}}, \omega\right) \\
& \hat{\mathbf{x}}_{\text {init }}=\operatorname{Initialize}\left(\mathbf{y}, \boldsymbol{\Phi}, \boldsymbol{\Psi}, \mathbf{x}_{t-1}, \omega\right) \\
& \hat{\mathbf{x}}_{\text {pred }}^{\prime}=\operatorname{MotionCompensationTelescopic}\left(\hat{\mathbf{x}}_{\text {init }}, \hat{\mathbf{X}}\right) \\
& \hat{\mathbf{x}}_{\text {pred }}^{\prime \prime}=\operatorname{MotionCompensation}\left(\hat{\mathbf{x}}_{\text {init }}, \hat{\mathbf{x}}_{t-1}\right) \\
& \hat{\mathbf{x}}_{\text {pred }}=\omega \hat{\mathbf{x}}_{\text {pred }}^{\prime}+(1-\omega) \hat{\mathbf{x}}_{\text {pred }}^{\prime \prime} \\
& \mathbf{y}_{\text {pred }}=\mathbf{\Phi} \hat{\mathbf{x}}_{\text {pred }} \\
& \mathbf{y}_{\mathrm{r}}=\mathbf{y}-\hat{\mathbf{y}}_{\text {pred }} \\
& \hat{\mathbf{x}}_{\mathrm{r}}=\operatorname{CS}-\operatorname{PL}\left(\mathbf{y}_{\mathrm{r}}, \boldsymbol{\Phi}, \boldsymbol{\Psi}\right) \\
& \hat{\mathbf{x}}_{t}=\hat{\mathbf{x}}_{\text {pred }}+\hat{\mathbf{x}}_{\mathrm{r}}
\end{aligned}
$$

Here, MotionCompensation $(\cdot)$ implements block-based singlereference-frame ME/MC, MotionCompensationTelescopic $(\cdot)$ is the telescopic-search ME/MC applied to the entire GOP, $\mathbf{y}_{t}$ is the set of measurements for the current frame, $\hat{\mathbf{x}}_{t-1}$ is the previously reconstructed preceding frame, $\hat{\mathbf{X}}$ is the set of $P$ previously reconstructed frames for the $P$-frame GOP that contains the current frame, and $\omega$ is a weighting factor. We note that the reconstruction of the current frame, $\hat{\mathbf{x}}_{t}$, produced by MC-CS-PL is placed into the GOP $\hat{\mathbf{X}}$, replacing the corresponding frame there, prior to the application of MC-CS-PL to the next frame.

As for weight $\omega$, we have found that restricting ME/MC to simply the preceding frame works well at low subrate; however, as the subrate increases, the telescopic search tends to work better. As a consequence, we balance the two forms of $\mathrm{ME} / \mathrm{MC}$ with weight $\omega(0 \leq \omega \leq 1)$ depending on the subrate of the non-key frames; i.e.,

$$
\omega= \begin{cases}0.2, & S_{\mathrm{NK}}<0.2 \\ 0.8, & S_{\mathrm{NK}}>0.5 \\ 2 S_{\mathrm{NK}}-0.2, & \text { otherwise }\end{cases}
$$

where $S_{\mathrm{NK}}$ is subrate for non-key frame ${ }^{2}$.

To obtain the initial reconstructions for the GOP, $\hat{\mathbf{X}}$, as needed to start MC-CS-PL, we apply the following procedure to produce each $\hat{\mathbf{x}}_{t}$ of the GOP:

$$
\begin{aligned}
& \text { function } \hat{\mathbf{x}}_{\text {init }}=\operatorname{Initialize}\left(\mathbf{y}, \boldsymbol{\Phi}, \boldsymbol{\Psi}, \mathbf{x}_{t-1}, \omega\right) \\
& \hat{\mathbf{x}}^{\prime}=\operatorname{CS}-\operatorname{PL}(\mathbf{y}, \boldsymbol{\Phi}, \boldsymbol{\Psi})
\end{aligned}
$$

\footnotetext{
${ }^{2}$ We fix $S_{\mathrm{K}}$, the subrate for the key frame that starts the GOP, to be 0.7 .
}

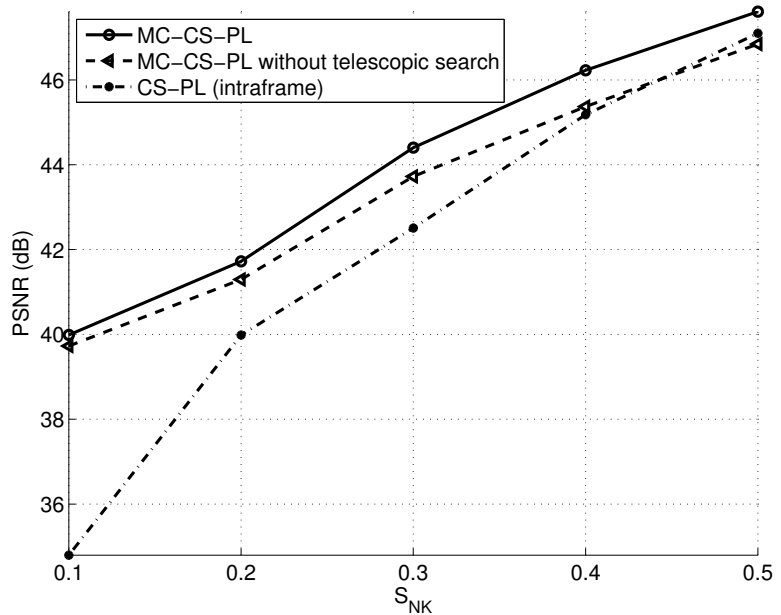

Figure 3: "Cardiac" for $S_{\mathrm{K}}=0.7$.

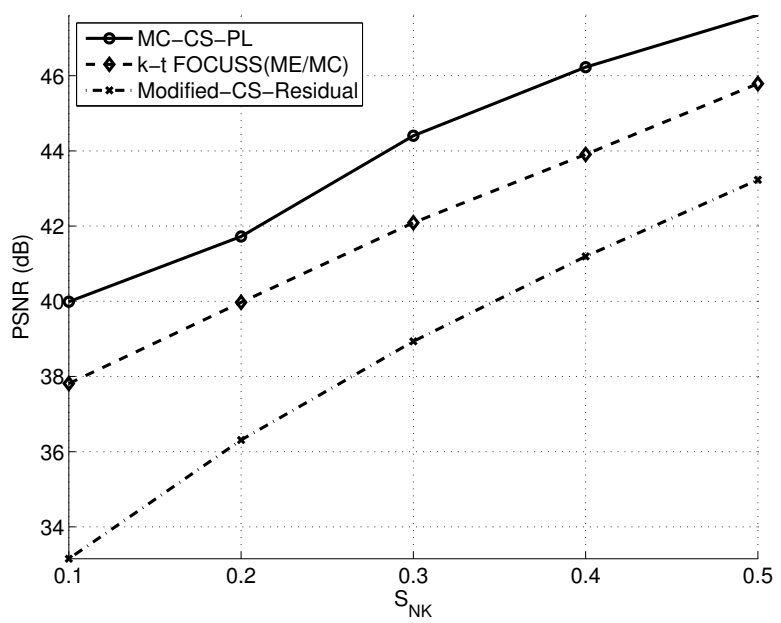

Figure 4: "Cardiac" for $S_{\mathrm{K}}=0.7$.

$$
\begin{aligned}
& \mathbf{y}_{\mathrm{r}}=\mathbf{y}-\mathbf{\Phi} \mathbf{x}_{t-1} \\
& \hat{\mathbf{x}}^{\prime \prime}=\operatorname{CS}-\operatorname{PL}\left(\mathbf{y}_{\mathrm{r}}, \boldsymbol{\Phi}, \boldsymbol{\Psi}\right)+\mathbf{x}_{\text {pred }} \\
& \hat{\mathbf{x}}_{\text {init }}=\omega \hat{\mathbf{x}}_{\text {init }}^{\prime}+(1-\omega) \hat{\mathbf{x}}_{\text {init }}^{\prime \prime}
\end{aligned}
$$

An initial reconstruction can be produced via 2D CS-PL or simple use of the previously reconstructed frame as a (non-ME/MC) prediction. Empirical results in [7] show that a weighted combination of both approaches outperforms either approach used alone. Here, $\omega$ is the same weight from (7).

Finally, we note that we have described the MC-CS-PL here in terms of forward motion prediction [23] wherein the current frame is predicted by a preceding frame. However, as described in [6,7], we can equally use backward prediction in which the current frame is predicted by a subsequent frame. Consequently, we implement MC-CS-PL using forward prediction for the first half of the GOP, backward prediction for the last half of the GOP, and both for the center frame. 

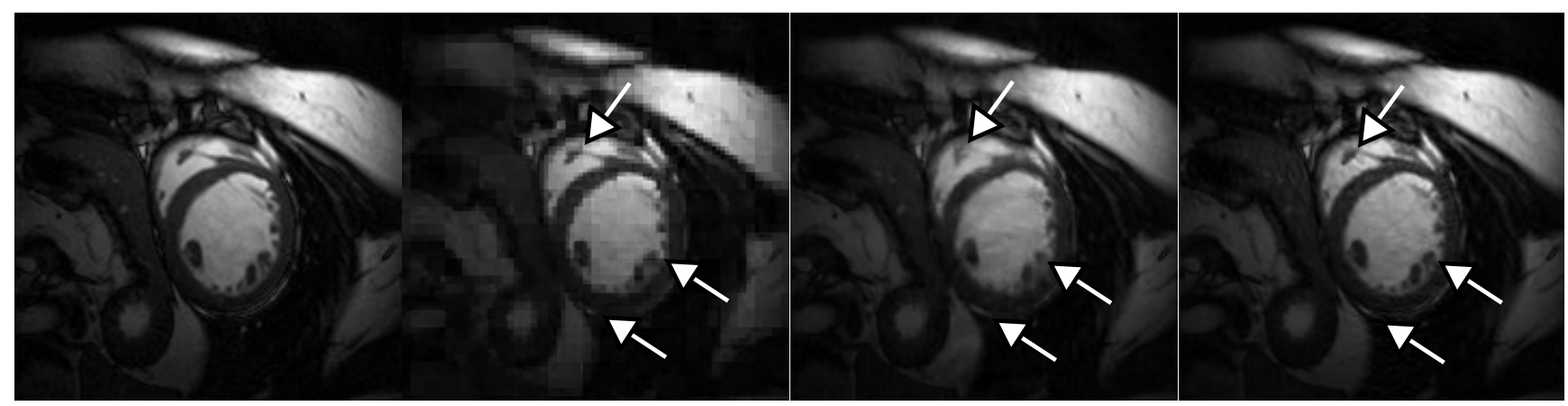

Figure 6: Frame 4 (center frame of first GOP) of "Cardiac" for $S_{\mathrm{K}}=0.7$, and $S_{\mathrm{NK}}=0.1$ (equivalently 10-time acceleration). Left to right: original frame, Modified-CS-Residual, k-t FOCUSS, and MC-CS-PL. All images are cropped for the detail.

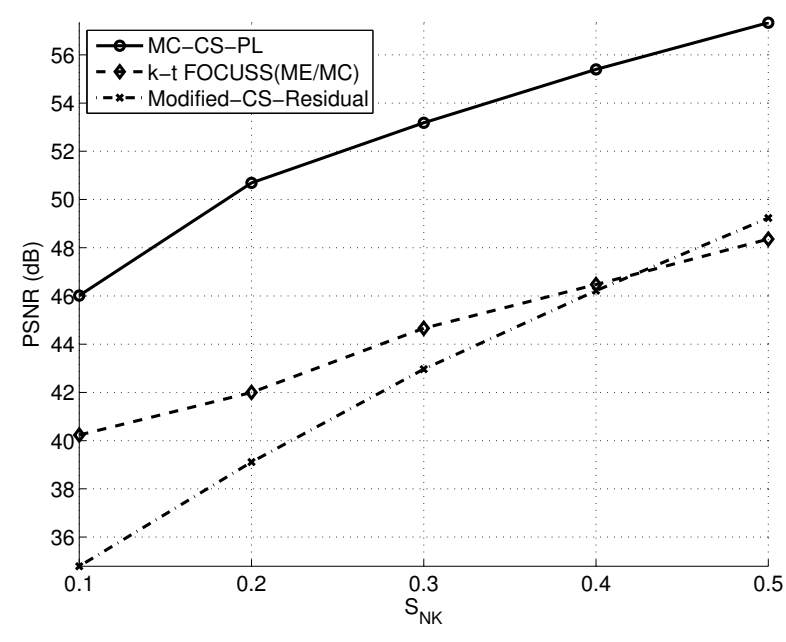

Figure 5: "Larynx" for $S_{\mathrm{K}}=0.7$.

\section{EXPERIMENTAL RESULTS}

We now examine the performance of MC-CS-PL reconstruction relative to a corresponding "intraframe" reconstruction to demonstrate that significant gain results from the explicit exploitation of motion information within the CS reconstruction of dynamic MRI. We also compare to two prominent CS reconstruction algorithms, Modified-CS-Residual [3] and k-t FOCUSS with ME/MC $[1,2]$. We use implementations of k-t FOCUSS ${ }^{3}$ and ModifiedCS-Residual $^{4}$ available from their respective authors.

We use the MRI sequences "Cardiac" (24 frames) and "Larynx" (56 frames). Both of sequences are grayscale frames with spatial size of $256 \times 256$. The sequences are subject to partial Fourier projection applied frame by frame; for MC-CS-PL, we use a radial sensing trajectory with uniformly spaced rays (as in $\ell_{1}$ MAGIC $^{5}$ ) for measurement operator $\boldsymbol{\Phi}$; for other techniques, we use the default measurement process used in the respective software implementations. In all cases, we use a GOP size of $P=8$ frames with key frames starting each GOP having a subrate of

\footnotetext{
${ }^{3}$ http://bisp.kaist.ac.kr/research_02.htm

${ }^{4}$ http://home.engineering. iastate.edu/ luwei/modcs/

${ }^{5}$ http://www.acm.caltech.edu/l1magic/
}

$S_{\mathrm{K}}=0.7$. The intervening non-key frames have subrate $S_{\mathrm{NK}}$ varying between 0.1 and 0.5 . As a primary measure of reconstruction quality, we calculate the PSNR averaged over all non-key frames under consideration. For the ME/MC process in MC-CS-PL, we use full-search ME with full-pixel accuracy, a block size of 32, a search window of \pm 7 pixels, and a telescopic search range within the respective GOP. We note that k-t FOCUSS uses an overlapped search, a block size of 2 , and a search window of \pm 7 by default in the original software package.

First, we compare MC-CS-PL with simpler variants of the same, namely "intraframe" reconstruction (i.e., CS-PL applied independently frame by frame) and MC-CS-PL without telescopic search. We see in Fig. 3 that both of the MC-CS-PL techniques achieve higher-fidelity reconstruction especially at low subrate, meaning that exploiting motion information enhances intraframe reconstruction. Additionally, the results show that the telescopic search results in improved performance at higher subrates.

Next, we compare MC-CS-PL to k-t FOCUSS as well as Modified-CS-Residual in Figs. 4 and 5. These results indicate that MCCS-PL with telescopic search outperforms the other techniques considered, sometimes by as much as $5-8 \mathrm{db}$. Finally, Fig. 6 illustrates sample visual results for the "Cardiac" sequence. Because Modified-CS-Residual inherently keeps only the most significant transform coefficients over the frames, it misses some detail DWT coefficients and thereby does not capture the contours of the organs well. On the other hand, k-t FOCUSS yields clear and sharp contours albeit with some blurring and smearing elsewhere. On the other hand, MC-CS-PL result appears to capture most detail of the original frame without significant distortion.

\section{CONCLUSIONS}

In this paper, we examined the use of $\mathrm{ME} / \mathrm{MC}$ in CS recovery of dynamic MRI. For 2D reconstruction, a directional transform and statistical thresholding are plugged into PL to capture the sparse pattern in the MRI while keeping directional features of the image. For ME/MC, we find that a spatially narrow search over a long temporal range results in better block matches in order to capture cyclic motion pattern due to the repetitive behavior of internal organ. Incorporating a telescopic motion search into CS-PL reconstruction, the resulting MC-CS-PL algorithm outperforms both k-t FOCUSS and Modified-CS-Residual, prior CS reconstruction techniques designed specifically for dynamic MRI. 


\section{REFERENCES}

[1] H. Jung, K. Sung, K. S. Nayak, E. Y. Kim, and J. C. Ye, "k-t FOCUSS: A general compressed sensing framework for high resolution dynamic MRI," Magnetic Resonance in Medicine, vol. 61, no. 1, pp. 103-116, January 2009.

[2] H. Jung and J. C. Ye, "Motion estimated and compensated compressed sensing dynamic magnetic resonance imaging: What we can learn from video compression techniques," Imaging Systems and Technology, vol. 20, no. 2, pp. 81-98, June 2010.

[3] N. Vaswani and W. Lu, "Modified-CS: Modifiying compressive sensing for problems with partially known support," IEEE Transactions on Signal Processing, vol. 58, no. 9, pp. 4595-4607, September 2010.

[4] N. Vaswani, "LS-CS-Residual (LS-CS): Compressive sensing on least squares residual," IEEE Transactions on Signal Processing, vol. 57, no. 8, pp. 4108-4120, August 2010.

[5] Ç. Bilen, Y. Wang, and I. W. Selesnick, "High-speed compressed sensing reconstruction in dynamic parallel MRI using augmented Lagrangian and parallel processing," IEEE Journal on Emerging and Selected Topics in Circuits and Systems, vol. 2, no. 3, pp. 370-379, September 2012.

[6] S. Mun and J. E. Fowler, "Residual reconstruction for blockbased compressed sensing of video," in Proceedings of the Data Compression Conference, J. A. Storer and M. W. Marcellin, Eds., Snowbird, UT, March 2011, pp. 183-192.

[7] J. E. Fowler, S. Mun, and E. W. Tramel, "Block-based compressed sensing of images and video," Foundations and Trends in Signal Processing, vol. 4, no. 4, pp. 297-416, March 2012.

[8] M. Trocan, E. W. Tramel, J. E. Fowler, and B. PesquetPopescu, "Compressed-sensing recovery of multiview image and video sequences using signal prediction," Multimedia Tools and Applications, to appear.

[9] W. Lu and N. Vaswani, "Modified compressive sensing for real-time dynamic MR imaging," in Proceedings of the International Conference on Image Processing, Cairo, Egypt, November 2009, pp. 3045-3048.

[10] S. Mun and J. E. Fowler, "Block compressed sensing of images using directional transforms," in Proceedings of the International Conference on Image Processing, Cairo, Egypt, November 2009, pp. 3021-3024.

[11] M. Lustig, D. L. Donoho, J. M. Santos, and J. M. Pauly, "Compressed sensing MRI," IEEE Signal Processing Magazine, vol. 25, no. 2, pp. 72-82, March 2008.

[12] L. Gan, "Block compressed sensing of natural images," in Proceedings of the International Conference on Digital Signal Processing, Cardiff, UK, July 2007, pp. 403-406.

[13] J. Haupt and R. Nowak, "Signal reconstruction from noisy random projections," IEEE Transactions on Information Theory, vol. 52, no. 9, pp. 4036-4048, September 2006.

[14] M. Bertero and P. Boccacci, Introduction to Inverse Problems in Imaging. Bristol, UK: Institute of Physics Publishing, 1998.

[15] T. Blumensath and M. E. Davies, "Iterative thresholding for sparse approximations," The Journal of Fourier Analysis and Applications, vol. 14, no. 5, pp. 629-654, December 2008.
[16] — - "Iterative hard thresholding for compressed sensing," Applied and Computational Harmonic Analysis, vol. 27, no. 3, pp. 265-274, November 2009.

[17] N. G. Kingsbury, "Complex wavelets for shift invariant analysis and filtering of signals," Journal of Applied Computational Harmonic Analysis, vol. 10, pp. 234-253, May 2001.

[18] L. Şendur and I. W. Selesnick, "Bivariate shrinkage functions for wavelet-based denoising exploiting interscale dependency," IEEE Transactions on Signal Processing, vol. 50, no. 11, pp. 2744-2756, November 2002.

[19] E. Candès, J. Romberg, and T. Tao, "Robust uncertainty principles: Exact signal reconstruction from highly incomplete frequency information," IEEE Transactions on Information Theory, vol. 52, no. 2, pp. 489-509, February 2006.

[20] C. Li, W. Yin, H. Jiang, and Y. Zhang, "An efficient augmented Lagrangian method with applications to total variation minimization," Department of Computational and Applied Mathematics, Rice University, Tech. Rep. TR12-13, 2012.

[21] J. Lee and B. W. Dickinson, "Temporally adaptive motion interpolation exploiting temporal masking in visual perception," IEEE Transactions on Image Processing, vol. 3, no. 5, pp. 513-526, September 1994.

[22] J. L. Mitchell, W. B. Pennebaker, C. E. Fogg, and D. J. LeGall, MPEG Video Compression Standard. New York, NY: Chapman \& Hall, 1996.

[23] M. Ghanbari, Standard Codecs: Image Compression to Advanced Video Coding. London, United Kingdom: The Institution of Electrical Engineers, 2003. 